\title{
CRITICAL MASS FOR MERGING IN DOUBLE WHITE DWARFS
}

\author{
Izumi HACHISU ${ }^{1}$ and Mariko KATO ${ }^{2}$ \\ ${ }^{1}$ Department of Aeronautical Engineering, Kyoto University \\ ${ }^{2}$ Department of Astronomy, Keio University
}

\section{I . INTRODUCTION}

We examine whether or not double white dwarfs are ultimately merging into one body. It has been argued that such a double white dwarf system forms from some intermediate-mass binary stars and will merge due to the gravitational radiation which decreases the separation of binary. After filling the inner critical Roche lobe, the less massive component begins to transfer its mass to the more massive one. When the mass transfer rate exceeds a some critical value, a common envelope is formed. If the common envelope is hydrostatic, the mass transfer is tuned up to be a some value which depends only on the white dwarf mass, radius, and the Roche lobe size. The mass transfer from the less massive to the more massive components leads the separation to increase. On the other hand, the gravitational radiation effect reduces the separation. Which effect wins determines the fate of double white dwarfs, that is, whether merging or not merging. Since the formula of the gravitational radiation effect is well known, we have studied the mass accretion rate in common envelope phase of double white dwarfs assuming the Roche lobe size is as small as $0.03 R_{\odot}$ or $0.1 R_{\odot}$.

\section{I CRITICAL MASS ACCRETION RATES IN COMMON ENVELOPE PHASE}

When the mass transfer exceeds the so-called Eddington accretion rate, i.e.,

$$
\dot{\mathrm{M}}_{\mathrm{Edd}}=\mathrm{L}_{\mathrm{Edd}} /\left[\mathrm{GM}_{W D} / \mathrm{R}_{\mathrm{WD}}-\mathrm{GM}_{W D} / \mathrm{R}_{\mathrm{ph}}\right] \text {, }
$$

where $L_{E d d}$ is the Eddington Iuminosity at the photosphere, i.e., $L_{E d d}=$ $4 \pi \mathrm{eGM}_{W D} / k$, the acereted envelope expands to overfill the Roche lobe. Two pressures between the extended envelope and the mass-losing white dwarf balances with each other. Hydrostatic balance is reached in the common envelope. We assume that the envelope around the more masive 
white dwarf just fills the same potential surface as the less massive one's surface. Assuming further that the envelope around the white dwarf is spherically symmetric, we have obtained the mass accretion rates.

The accreting matter releases the gravitational energy. In the equation of energy conservation, we include the homologous term, i.e.,

$$
\frac{d L_{r}}{d M_{r}}=\varepsilon_{g}^{(h)}+\varepsilon_{\mathrm{n}},
$$

where $M_{r}$ is the mass within the radius $\underline{r}, \varepsilon_{g}^{(h)}$ the homologous term of $\varepsilon_{g}$ (e.g., Sugimoto and Nomoto 1975; Nariai, Nomoto, and sugimoto $1980), \varepsilon$ n the nuclear energy generation per unit time and per unit mass. We implicitly assume that the entropy of the transferred matter is the same as the entropy of at the top of the extended envelope.

It is found that there is the minimum envelope mass for a given set of the white dwarf mass and the Roche lobe size. For the minimum envelope mass, the mass accretion rate is very close to the Eddington accretion rate defined by equation (1). The mass accretion rate decreases gradually until helium/carbon is ignited at the bottom of the envelope as the envelope mass is increased. For $\mathrm{M}_{W D} 20.8 \mathrm{M}_{\odot}$, however, helium is ignited before the envelope expands to $0.03 \mathrm{R}_{\odot}$ if the accreted matter is helium. For $M_{W D}>1.1 \mathrm{M}_{\odot}$, carbon is ignited off-center before the envelope expands to $0.03 R_{\odot}$ if the matter is carbon-oxygen mixture. The mass accretion rates are plotted in Figures 1 and 2 .

\section{I I. CRITICAL MASS FOR MERGING}

\section{a) $\mathrm{He}-\mathrm{CO}$ pa i $\mathrm{r}$}

When the white dwarf mass is larger than $0.8 \mathrm{M}_{\odot}$, helium is ignited before a common envelope is formed. After helium ignition, the structure of the envelope changes and the envelope expands to overfill the Roche lobe. Then a common envelope may be formed around the systern. Then the mass transfer rate is given by the steady burning line in Figure $2 \mathrm{a}$. The change in the separation may be determined by

$$
\frac{\dot{a}}{a}=-2(1-q) \frac{\dot{M}_{2}}{M_{2}}+\frac{2}{J}\left(\frac{d J}{d t}\right)_{G R}=\tau_{M}{ }^{-1}-\tau_{G}{ }^{-1},
$$

if the total mass is conserved, where $\dot{a}$ is the increasing rates of the separation, $\dot{M}_{2}$ the mass transfer rates, and the second term in the middle means the decrease in the separation due to the loss of the orbital angular momentum by gravitational wave radiation, i.e., 


$$
\frac{1}{J}\left(\frac{\mathrm{dJ}}{\mathrm{dt}}\right)_{\mathrm{GR}}=-1.94 \times 10^{-2}\left(\frac{\mathrm{M}_{1}}{\mathrm{M}_{\odot}}\right)^{3}(1+\mathrm{q}) \mathrm{q}\left(\mathrm{a} / 10^{9} \mathrm{~cm}\right)^{-4} \mathrm{y} \mathrm{r}^{-1}
$$

It is clear that if the time scale of the mass transfer $\tau_{M}$ is shorter than that of the gravitational wave radiation $\tau_{G}$, two white dwarfs are separating from each other. If $\tau_{M}>\tau_{G}$, two stars are merging into one body.

Equating $\tau_{M}=\tau_{G}$, we obtain the critical masses for merging in common envelope phase as:

$$
\mathrm{M}_{\mathrm{er}}(\mathrm{He}) \sim 0.25 \mathrm{M}_{\odot} \text { for } \mathrm{M}_{\mathrm{CO}} \sim 1 \mathrm{M}_{\odot} \text {. }
$$

b) $\underline{\mathrm{CO}-\mathrm{CO}}$ or $\mathrm{CO}+\mathrm{ONeMg}$ pair

Two white dwarfs are always merging into one body because $\tau_{G}{ }^{>}$ $\tau$ M always holds.

\section{c) $\mathrm{He}-\mathrm{He}$ pair}

When the total mass of the system is larger than $\sim 0.6 \mathrm{M}_{\odot}$, two white dwarfs are probably merging after helium is ignited off-center and the mass accreting helium white dwarfs expands to a few solar radi (Saio and Nomoto, private communication). The total mass is less than $0.6 M_{\odot}$ and the less massive white dwarf mass is smaller than $0.2 M_{\odot}$, two helium white dwarfs will not merge.

\section{REFERENCES}

Hachisu, I., and Kato, M. 1988, Ap. J., submitted.

Kato, M., Saio, H., and Hachisu, I. 1988, Ap. J., submitted.

Nariai, K., Nomoto, K., Sugimoto, D., 1980 Pub. Astr. Soc. Japan., 32, 473.

Sugimoto, D., and Nomoto, K. 1975, Publ. Astr. Soc. Japan, 27, 197.

\section{figure captions}

Fig. 1-The mass accretion rate is plotted against the envelope mass for various white dwarf core masses. Open triangles: maximum accretion rate in a static envelope. Open circles: mass accretion rate at the helium ignition. Filled circles: mass accretion (i.e., steady burning) rate of helium matter. Numbers attached are the core mass of white dwarfs in solar mass units. $R_{p h}=0.1 R_{\odot}$ is assumed.

Fig. 2-The mass accretion rates at the maximum accretion (denoted by "max"), at the helium ignition (denoted by "ignition"), and at the steady helium shell burning are plotted against the core mass for two cases of the Roche lobe sizes, i.e., $0.1 R_{\odot}$ and $0.03 R_{\odot}$ for (a) helium accretion and for (b) C+O accretion. 


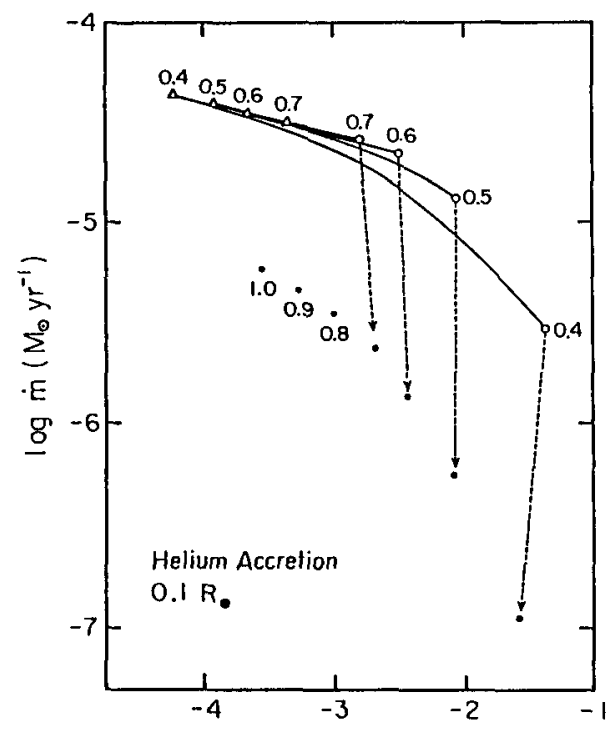

$\log \Delta M / M_{\odot}$

FIG. I

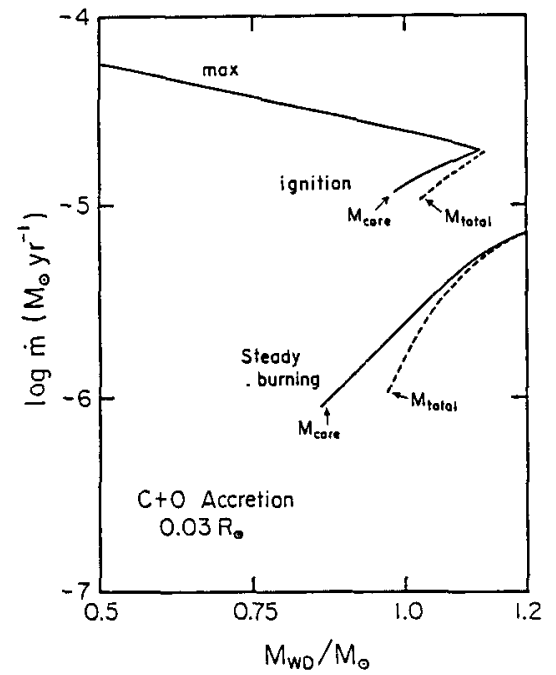

FIG.2b

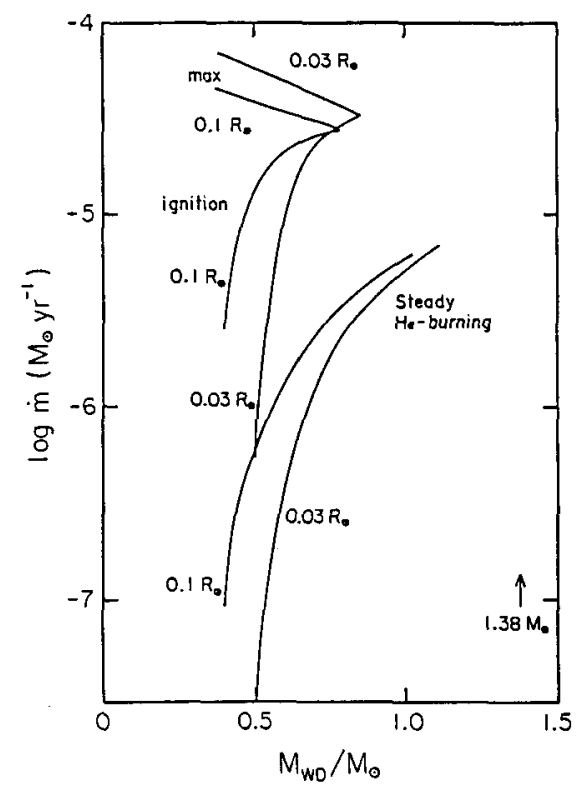

FIG. 2 a 